\title{
The maximal number of runs in standard Sturmian words
}

\author{
Paweł Baturo ${ }^{\ddagger}$ Marcin Piątkowski ${ }^{* *}$ Wojciech Rytter $\sharp \sharp^{\sharp}$ \\ $\ddagger$ Faculty of Mathematics and Computer Science \\ Nicolaus Copernicus University \\ $\sharp$ Institute of Informatics \\ Warsaw University \\ \{pbaturo, marcin.piatkowski, rytter\}@mat.umk.pl
}

Submitted: Jun 25, 2012; Accepted: Jan 7, 2013; Published: Jan 21, 2013

Mathematics Subject Classification: 05C88

\begin{abstract}
We investigate some repetition problems for a very special class $\mathcal{S}$ of strings called the standard Sturmian words, which have very compact representations in terms of sequences of integers. Usually the size of this word is exponential with respect to the size of its integer sequence, hence we are dealing with repetition problems in compressed strings. An explicit formula is given for the number $\rho(w)$ of runs in a standard word $w$. We show that $\frac{\rho(w)}{|w|} \leqslant \frac{4}{5}$ for each $w \in \mathcal{S}$, and there is an infinite sequence of strictly growing words $w_{k} \in \mathcal{S}$ such that $\lim _{k \rightarrow \infty} \frac{\rho\left(w_{k}\right)}{\left|w_{k}\right|}=\frac{4}{5}$. Moreover, we show how to compute the number of runs in a standard Sturmian word in linear time with respect to the size of its compressed representation.
\end{abstract}

\section{Introduction}

A run (a maximal repetition) is a non-extendable (with the same period) periodic segment in a string, in which the period repeats at least twice. Runs are important in combinatorics on words and many practical applications: data compression, computational biology, pattern-matching and so on. The structure of repetitions is almost completely understood

${ }^{*}$ The study is cofounded by the European Union from resources of the European Social Fund. Project PO KL "Information technologies: Research and their interdisciplinary applications", Agreement UDAPOKL.04.01.01-00-051/10-00.

†Supported by the grant N206 566740 of the National Science Center 
for the class of Fibonacci words, see [11], [17], [9]. In this paper we investigate the structure of runs in class $\mathcal{S}$ of standard Sturmian words and give an exact formula and a tight asymptotic bound for the number of maximal repetitions.

We continue here the work of [6], where it was shown how to compute the number of runs for block-complete Sturmian words (not all standard Sturmian words have this property) in linear time with respect to the size of the whole word. We show also the algorithm, which computes the number of runs in any standard word in linear time with respect to the size of its compressed representation (the directive sequence), hence in logarithmic time with respect to the length of the word.

Throughout the paper we use the standard notions of combinatorics on words. In particular, words are finite sequences over a finite set $\Sigma$ of letters, called the alphabet. For a word $w=w_{1} w_{2} \ldots w_{n}$, by $w[i]$ we denote its $i$-th letter (namely $w_{i}$ ), by $w[i . . j]$ the subword $w_{i} w_{i+1} \ldots w_{j}$, by $|w|$ its length and by $|w|_{a}$ the number of letters $a$ occurring in $w$.

The number $i$ is a period of the word $w$ if $w[j]=w[i+j]$ for all $i$ with $i+j \leqslant|w|$. The minimal period of $w$ will be denoted by $\operatorname{period}(w)$. We say that a word $w$ is periodic if $\operatorname{period}(w) \leqslant \frac{|w|}{2}$. A word $w$ is said to be primitive if $w$ is not of the form $z^{k}$, where $z$ is a nonempty word and $k \geqslant 2$ is a natural number.

A maximal repetition (a run, in short) in a word $w$ is an interval $\alpha=[i . . j]$, such that $w[i . . j]=u^{k} v(k \geqslant 2)$ is a nonempty periodic subword of $w$, where $u$ is of the minimal length and $v$ is a proper prefix (possibly empty) of $u$, that can not be extended (neither $w[i-1 . . j]$ nor $w[i . . j+1]$ is a run with the period $|u|)$.

A run $\alpha$ can be properly included as an interval in another run $\beta$, but in this case $\operatorname{period}(\alpha)<\operatorname{period}(\beta)$. The value of the $\operatorname{run} \alpha=[i \ldots j]$ is the factor $\operatorname{val}(\alpha)=w[i \ldots j]$. When it makes no ambiguity we identify sometimes run with its value and the period of the run $\alpha=[i \ldots j]$ with the subword $w[i . . p e r i o d(w)]$, called also the generator of the repetition. The meaning will always be clear from the context. Observe that two different runs could correspond to the identical subwords, if we disregard their positions. Hence runs are also called the maximal positioned repetitions.

Example 1. Let $w=$ ababaabababaabababaabababaababaab.

There are 5 runs with the period $a$ :

$$
w[5 . .6]=a^{2}, \quad w[12 . .13]=a^{2}, \quad w[19 . .20]=a^{2}, \quad w[26 . .27]=a^{2}, \quad w[31 . .32]=a^{2},
$$

5 runs with the period $a b$ :

$$
\begin{aligned}
w[1 . .5] & =(a b)^{2} a, \quad w[6 . .12]=(a b)^{3} a, \quad w[13 . .19]=(a b)^{3} a, \\
w[20 . .26] & =(a b)^{3} a, \quad w[27 . .31]=(a b)^{2} a
\end{aligned}
$$

4 runs with the period aba:

$$
w[3 . .8]=(a b a)^{2}, \quad w[10 . .15]=(a b a)^{2}, \quad w[17 . .22]=(a b a)^{2}, \quad w[24 . .29]=(a b a)^{2},
$$


4 runs with the period ababa:

$$
\begin{aligned}
w[1 . .10] & =(a b a b a)^{2}, & w[8 . .17] & =(a b a b a)^{2}, \\
w[15 . .24] & =(a b a b a)^{2}, & w[22 . .33] & =(a b a b a)^{2} a b,
\end{aligned}
$$

and 1 run with the period ababaab: $w[1 . .31]=(a b a b a a b)^{4} a b a$. Alltogether we have 19 runs, see Figure 1 for comparison.

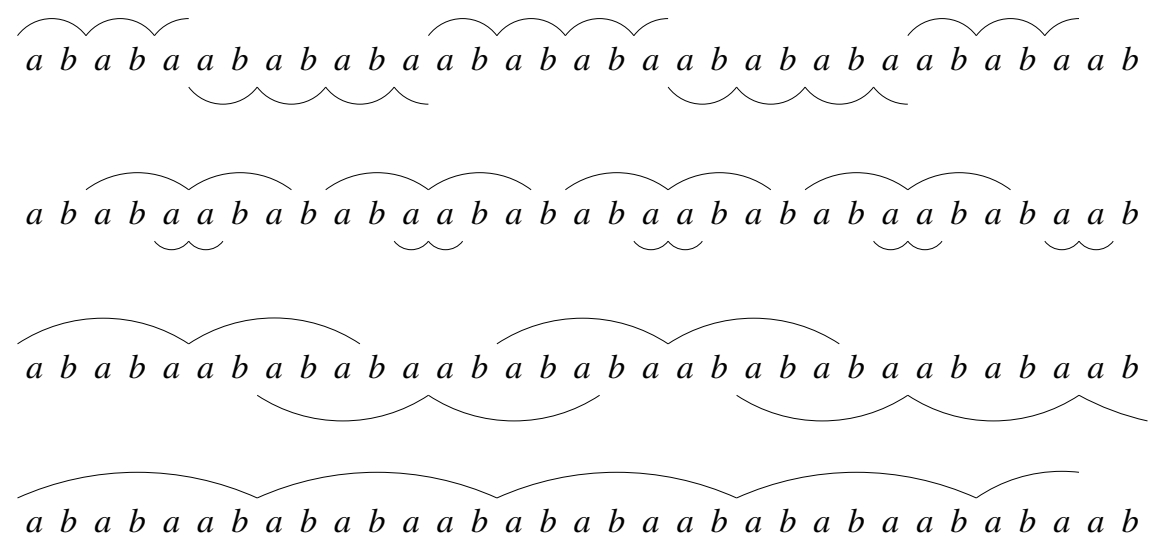

Figure 1: The structure of maximal repetitions for the example binary word.

Denote by $\rho(w)$ the number of runs in the word $w$ and by $\rho(n)$ the maximal number of runs in the words of length $n$. The most interesting related open conjecture is:

$$
\rho(n)<n \text {. }
$$

In 1999 Kolpakov and Kucherov (see [10]) showed that the number $\rho(w)$ of runs in a string $w$ is $O(|w|)$, but the exact multiplicative constant coefficient is still unknown. The best known results related to the value of $\rho(n)$ are

$$
0.944542 n \leqslant \rho(n) \leqslant 1.048 n \text {. }
$$

The upper bound is by [3], [4] and the lower bound is by [7], [8], [12]. Table 1 (at the end of this paper) shows the maximal number of runs and the repetition ratio in all binary words compared to standard words (to be defined in the next section) for small $n$.

\section{Standard words}

A directive sequence is an integer sequence: $\gamma=\left(\gamma_{0}, \gamma_{1}, \ldots, \gamma_{n}\right)$, where $\gamma_{0} \geqslant 0$ and $\gamma_{i}>0$ for $i=1,2, \ldots, n$. The standard word corresponding to $\gamma$, denoted by $x_{n+1}=\operatorname{Sw}(\gamma)$, is described by the recurrences of the form:

$$
x_{-1}=b, \quad x_{0}=a, \quad \ldots, \quad x_{n}=\left(x_{n-1}\right)^{\gamma_{n-1}} x_{n-2}, \quad x_{n+1}=\left(x_{n}\right)^{\gamma_{n}} x_{n-1} .
$$


The sequence of words $\left\{x_{i}\right\}_{i=0}^{n+1}$ is called a standard sequence. Every word occurring in a standard sequence is a standard word, and every standard word occurs in some standard sequence. We assume that the standard word given by the empty directive sequence is the single letter $a$ and $\operatorname{Sw}(0)=b$. The class of all standard words is denoted by $\mathcal{S}$.

Example 2. Consider the directive sequence $\gamma=(1,2,1,3,1)$. Then $\operatorname{Sw}(1,2,1,3,1)=x_{5}$, where:

$$
\begin{aligned}
& x_{-1}=b ; \quad x_{0}=a ; \\
& x_{1}=\left(x_{0}\right)^{1} \cdot x_{-1}=a \cdot b ; \\
& x_{2}=\left(x_{1}\right)^{2} \cdot x_{0}=a b \cdot a b \cdot a ; \\
& x_{3}=\left(x_{2}\right)^{1} \cdot x_{1}=a b a b a \cdot a b ; \\
& x_{4}=\left(x_{3}\right)^{3} \cdot x_{2}=a b a b a a b \cdot a b a b a a b \cdot a b a b a a b \cdot a b a b a ; \\
& x_{5}=\left(x_{4}\right)^{1} \cdot x_{3}=a b a b a a b a b a b a a b a b a b a a b a b a b a \cdot a b a b a a b .
\end{aligned}
$$

For $\gamma_{0}>0$ we have standard words starting with the letter $a$ and for $\gamma_{0}=0$ we have standard words starting with the letter $b$. In fact the word $\operatorname{Sw}\left(0, \gamma_{1}, \ldots, \gamma_{n}\right)$ can be obtained from $\operatorname{Sw}\left(\gamma_{1}, \ldots, \gamma_{n}\right)$ by switching the letters $a$ and $b$.

Observe that for even $n>0$ the standard word $x_{n}$ has the suffix $b a$, and for odd $n>0$ it has the suffix $a b$. Moreover, every standard word consists either of repeated occurrences of the letter $a$ separated by single occurrences of the letter $b$ or repeated occurrences of the letter $b$ separated by single occurrences of the letter $a$. Those letters are called respectively the repeating letter and the single letter. If the repeating letter is a (letter $b$ respectively), the word is called the Sturmian word of the type $a$ (type $b$ respectively), see Definition 6.1.4 in [16] for comparison.

Without loss of generality we consider in this paper the standard Sturmian words of the type $a$, therefore we assume that $\gamma_{0}>0$. The words of the type $b$ can be considered similarly and all the results hold. For more interesting facts related to combinatorial structure of standard words see for instance in [1], [2], [13], [14] and [15].

Remark 3. Standard words are a generalization of Fibonacci words, the well known family of strings. By definition, Fibonacci words are standard words given by directive sequences of the form $\gamma=(1,1, \ldots, 1)$.

\section{Morphic reduction of standard words}

The recurrent definition of standard words from the previous section leads to a simple characterization by a composition of morphisms. Let $\gamma=\left(\gamma_{0}, \gamma_{1}, \ldots, \gamma_{n}\right)$ be a directive sequence. We associate with $\gamma$ a sequence of morphisms $\left\{h_{i}\right\}_{i=0}^{n}$, defined as:

$$
h_{i}:\left\{\begin{array}{l}
a \longrightarrow a^{\gamma_{i}} b \\
b \longrightarrow a
\end{array} \quad \text { for } 0 \leqslant i \leqslant n .\right.
$$




\section{Lemma 4.}

For $0 \leqslant i \leqslant n$ the morphism $h_{i}$ transforms a standard word into another standard word, and we have:

$$
\begin{aligned}
& \operatorname{Sw}\left(\gamma_{n}\right)=h_{n}(a), \\
& \operatorname{Sw}\left(\gamma_{i}, \gamma_{i+1}, \ldots, \gamma_{n}\right)=h_{i}\left(\operatorname{Sw}\left(\gamma_{i+1}, \gamma_{i+2}, \ldots, \gamma_{n}\right)\right)
\end{aligned}
$$

Proof. We prove the lemma by the induction on the length of the directive sequence.

Recall that the standard word given by the empty directive sequence is $a$. For $|\gamma|=1$ we have, by definition of standard words and the morphism $h_{n}$,

$$
\operatorname{Sw}\left(\gamma_{n}\right)=a^{\gamma_{n}} b=h_{n}(a)
$$

Assume now that $|\gamma|=k \geqslant 2$ and for directive sequences shorter than $k$ the statement holds. Then we have:

$$
\begin{aligned}
\operatorname{Sw}\left(\gamma_{i}, \ldots, \gamma_{n}\right) & =\left[\operatorname{Sw}\left(\gamma_{i}, \ldots, \gamma_{n-1}\right)\right]^{\gamma_{n}} \cdot \operatorname{Sw}\left(\gamma_{i}, \ldots, \gamma_{n-2}\right) \\
& \stackrel{i n d .}{=}\left[h_{i}\left(\operatorname{Sw}\left(\gamma_{i+1}, \ldots, \gamma_{n-1}\right)\right)\right]^{\gamma_{n}} \cdot h_{i}\left(\operatorname{Sw}\left(\gamma_{i+1}, \ldots, \gamma_{n-2}\right)\right) \\
& =h_{i}\left(\left[\operatorname{Sw}\left(\gamma_{i+1}, \ldots, \gamma_{n-1}\right)\right]^{\gamma_{n}} \cdot \operatorname{Sw}\left(\gamma_{i+1}, \ldots, \gamma_{n-2}\right)\right) \\
& =h_{i}\left(\operatorname{Sw}\left(\gamma_{i+1}, \ldots, \gamma_{n}\right)\right)
\end{aligned}
$$

and this concludes the proof.

Remark 5. As a direct corollary to Lemma 4 we have that for the directive sequence $\gamma=\left(\gamma_{0}, \gamma_{1}, \ldots, \gamma_{n}\right)$ :

$$
\operatorname{Sw}\left(\gamma_{0}, \gamma_{1}, \ldots, \gamma_{n}\right)=h_{0} \circ h_{1} \circ \ldots \circ h_{n}(a)
$$

Example 6. Consider the directive sequence $\gamma=(1,2,1,3,1)$.

We have (compare with Example 2):

$$
\begin{array}{rlrl}
\operatorname{Sw}(1) & =h_{4}(a) & & =a b \\
\operatorname{Sw}(3,1) & =h_{3}(\operatorname{Sw}(1)) & & =a a a b a \\
\operatorname{Sw}(1,3,1) & =h_{2}(\operatorname{Sw}(3,1)) & & \text { abababaab } \\
\operatorname{Sw}(2,1,3,1) & =h_{1}(\operatorname{Sw}(1,3,1)) & & \text { aabaaabaaabaaabaaba } \\
\operatorname{Sw}(1,2,1,3,1) & =h_{0}(\operatorname{Sw}(2,1,3,1)) & =\text { ababaabababaabababaabababaababaab. }
\end{array}
$$




\section{Reduction sequence}

Observe that the inverse morphism $h_{i}^{-1}$ can be seen as a reduction of the word $w_{i}=\operatorname{Sw}\left(\gamma_{i}, \ldots, \gamma_{n}\right)$ to $w_{i+1}=\operatorname{Sw}\left(\gamma_{i+1}, \ldots, \gamma_{n}\right)$. We utilise this approach to reduce the computation of runs in $w_{i}$ to the same computation in $w_{i+1}$. Our concept is similar to the one shown in [6], but is more closely related to the combinatorial structure of standard words.

Recall that $|w|_{a}$ denotes the number of occurrences of the letters $a$ in the word $w$. In the rest of this paper we use the following notation:

$$
\begin{aligned}
& N_{\gamma}(k)=\left|\operatorname{Sw}\left(\gamma_{k}, \gamma_{k+1}, \ldots, \gamma_{n}\right)\right|_{a}, \\
& M_{\gamma}(k)=\left|\operatorname{Sw}\left(\gamma_{k}, \gamma_{k+1}, \ldots, \gamma_{n}\right)\right|_{b},
\end{aligned}
$$

which enables us to simplify the formulas for the number of runs.

Remark 7 . The above definition, together with equations (1) and (2), implies:

$$
\begin{aligned}
N_{\gamma}(k) & =\gamma_{k} N_{\gamma}(k+1)+N_{\gamma}(k+2), \\
M_{\gamma}(k) & =N_{\gamma}(k+1)
\end{aligned}
$$

Remark 8. Observe that for Fibonacci word $F_{n}$ the number of the letters $a$ in $F_{n}$ equals the length of the word $F_{n-1}$, and therefore

$$
N_{\gamma}(k)=\left|F_{n-k-1}\right| \quad \text { and } \quad M_{\gamma}(k)=\left|F_{n-k-2}\right| .
$$

Example 9. Let $\gamma=(1,2,1,3,1)$ be a directive sequence. Then we have:

\begin{tabular}{r|l|l|l}
$\gamma$ & $\operatorname{Sw}(\gamma)$ & $|\operatorname{Sw}(\gamma)|_{a}$ & $|\operatorname{Sw}(\gamma)|_{b}$ \\
\hline$(1)$ & $a b$ & $N_{\gamma}(4)=1$ & $M_{\gamma}(4)=1$ \\
$(3,1)$ & aaaba & $N_{\gamma}(3)=4$ & $M_{\gamma}(3)=1$ \\
$(1,3,1)$ & abababaab & $N_{\gamma}(2)=5$ & $M_{\gamma}(2)=4$ \\
$(2,1,3,1)$ & aabaaabaaabaaabaaba & $N_{\gamma}(1)=14$ & $M_{\gamma}(1)=5$ \\
$(1,2,1,3,1)$ & ababaabababaabababaabababaababaab & $N_{\gamma}(0)=19$ & $M_{\gamma}(0)=14$
\end{tabular}

The following lemma enables to express the length of any standard word in terms of the numbers $N_{\gamma}(k)$ and $M_{\gamma}(k)$.

Lemma 10.

Let $w=\operatorname{Sw}\left(\gamma_{0}, \gamma_{1}, \ldots, \gamma_{n}\right), A=N_{\gamma}(2)$ and $B=N_{\gamma}(3)$. Then

$$
|w|=\left(\left(\gamma_{0}+1\right) \gamma_{1}+1\right) A+\left(\gamma_{0}+1\right) B .
$$


Proof. By the definition of $N_{\gamma}(k)$ and $M_{\gamma}(k)$ we have

$$
|w|=N_{\gamma}(0)+M_{\gamma}(0) \quad \text { and } \quad M_{\gamma}(0)=N_{\gamma}(1) .
$$

By repeated application of the formulas from Equation (3) we obtain:

$$
\begin{aligned}
|w| & =N_{\gamma}(0)+N_{\gamma}(1) \\
& =\left(\gamma_{0}+1\right) N_{\gamma}(1)+N_{\gamma}(2) \\
& =\left(\left(\gamma_{0}+1\right) \gamma_{1}+1\right) N_{\gamma}(2)+\left(\gamma_{0}+1\right) N_{\gamma}(3) \\
& =\left(\left(\gamma_{0}+1\right) \gamma_{1}+1\right) A+\left(\gamma_{0}+1\right) B
\end{aligned}
$$

and the proof is complete.

\section{The formula and the algorithm}

In this section we present the formula for the number of runs. The proof of its correctness is presented later in Section 6. We begin with the definition of some useful zero-one functions for testing the parity of a nonnegative integer $i$ :

$$
\operatorname{even}(i)=\left\{\begin{array}{ll}
1 & \text { for even } i \\
0 & \text { for odd } i
\end{array} \quad \text { and } \quad \operatorname{odd}(i)= \begin{cases}1 & \text { for odd } i \\
0 & \text { for even } i\end{cases}\right.
$$

and for testing if a positive integer $i$ equals 1 :

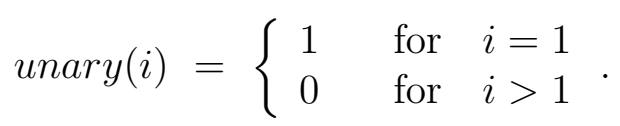

Theorem 11 (Formula for the number of runs in standard words).

Let $\gamma=\left(\gamma_{0}, \ldots, \gamma_{n}\right)$ be a directive sequence and $n \geqslant 3$. The number of runs in a standard word $w=\operatorname{Sw}\left(\gamma_{0}, \ldots, \gamma_{n}\right)$ is given by the following formula:

$$
\rho(w)= \begin{cases}2 A+2 B+\Delta(\gamma)-1 & \text { for } \gamma_{0}=\gamma_{1}=1 \\ \left(\gamma_{1}+2\right) A+B+\Delta(\gamma)-\operatorname{odd}(n) & \text { for } \gamma_{0}=1 ; \gamma_{1}>1 \\ 2 A+3 B+\Delta(\gamma)-\operatorname{even}(n) & \text { for } \gamma_{0}>1 ; \gamma_{1}=1 \\ \left(2 \gamma_{1}+1\right) A+2 B+\Delta(\gamma) & \text { for } \gamma_{0}>1 ; \gamma_{1}>1\end{cases}
$$

where: $A=N_{\gamma}(2), B=N_{\gamma}(3)$ and $\Delta(\gamma)=n-1-\left(\gamma_{1}+\ldots+\gamma_{n}\right)-\operatorname{unary}\left(\gamma_{n}\right)$. 
The detailed proof of the above theorem is shown in Section 6. The formula presented in Theorem 11 leads to a simple and fast algorithm for computation of the number of runs in standard words.

\section{Theorem 12.}

We can count the number of runs in any standard word $\operatorname{Sw}\left(\gamma_{0}, \ldots, \gamma_{n}\right)$ in linear time with respect to the length of the directive sequence $|\gamma|$.

Proof. The formula for the number of runs in standard words from Theorem 11 depends directly on the components of the directive sequence $\gamma=\left(\gamma_{0}, \ldots, \gamma_{n}\right)$ and the numbers $N_{\gamma}(2)$ and $N_{\gamma}(3)$. It is sufficient to prove that we can compute the numbers $N_{\gamma}(k)$ for $k=1,2,3$ in time $O(n)$. For this purpose we can iterate Equation (1):

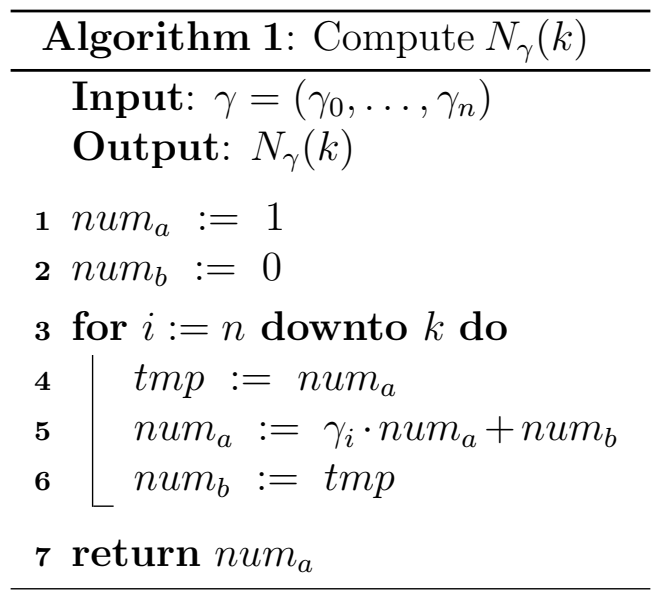

The correctness of the algorithm follows from the following invariant which holds after each iteration of the main for-loop:

num $_{a}$ equals $N_{\gamma}(i)$ (namely the number of letters $a$ in $\operatorname{Sw}\left(\gamma_{i}, \ldots, \gamma_{n}\right)$ );

num $_{b}$ equals $M_{\gamma}(i)=N_{\gamma}(i+1)$ (namely the number of letters $b \operatorname{in} \operatorname{Sw}\left(\gamma_{i}, \ldots, \gamma_{n}\right)$ ).

We start the algorithm with the empty directive sequence describing the word $\operatorname{Sw}(\emptyset)=a$, hence the initial values of the variables are: num $_{a}=1$ and num $_{b}=0$.

In each iteration of the for-loop values of $N_{\gamma}(i)$ and $M_{\gamma}(i)$ are computed from $N_{\gamma}(i+1)$ and $M_{\gamma}(i+1)$ by a direct application of Equation (4). Therefore, the loop computes consecutively values: $\left(N_{\gamma}(n), M_{\gamma}(n)\right),\left(N_{\gamma}(n-1), M_{\gamma}(n-1)\right), \ldots,\left(N_{\gamma}(k), M_{\gamma}(k)\right)$. The algorithm performs $n-k+1$ iterations of the for loop to compute $N_{\gamma}(k)$, hence its time complexity is $O(n)$, where $n$ denotes the length of the directive sequence $\gamma$.

Using Algorithm 1 and applying the formula from Equation (5) we can count the number of runs in any standard word in linear time with respect to the size of the directive sequence (logarithmic with respect to the length of the whole word). 
Now we can use the formula from Equation (5) to compute the number of runs in some example standard words.

Example 13. Let $\gamma=(1,2,1,3,1)$ be a directive sequence. We have $n=4$ and

$$
\operatorname{Sw}(\gamma)=\text { ababaabababaabababaabababaababaab. }
$$

In this case

$$
A=N_{\gamma}(2)=5, \quad B=N_{\gamma}(3)=4, \quad \Delta=(4-1)-7-1=-5, \quad \text { odd }(4)=0 .
$$

Theorem 11 implies:

$$
\begin{aligned}
\rho(w) & =\left(\gamma_{1}+2\right) A+B+\Delta-\operatorname{odd}(4) \\
& =4 A+B-5 \\
& =19
\end{aligned}
$$

see Figure 1 and Example 1 for comparison.

It is known that the number of runs in the $n$-th Fibonacci word $F_{n}$ is given by the formula

$$
\rho\left(F_{n}\right)=2 F_{n-2}+3
$$

see [11] for the proof. As the next example we derive this formula using Theorem 11.

Example 14. Recall that $F_{n}=\operatorname{Sw}(1,1, \ldots 1)$ ( $n$ ones) and in this case $N_{\gamma}(k)=F_{n-k-1}$. According to the formula from Theorem 11 we have:

$$
\begin{aligned}
\rho\left(F_{n}\right) & =2 N_{\gamma}(2)+2 N_{\gamma}(3)+n-1-n-1-1 \\
& =2 F_{n-3}+2 F_{n-4}-3 \\
& =2 F_{n-2}-3 .
\end{aligned}
$$

\section{Asympthotic behaviour of the number of runs}

The following lemma gives the bound for the number of runs in standard words described by the directive sequences of the length at most 2 .

Lemma 15 (Estimation for short $\gamma$ ). Let $\gamma=\left(\gamma_{0}, \ldots, \gamma_{n}\right)$ be a directive sequence, $w=\operatorname{Sw}(\gamma)$ and $n \leqslant 2$. Then $\rho(w)<\frac{4}{5}|w|$.

Proof. Recall that the standard word given by the empty directive sequence is $a$ and does not include any repetition. Therefore, we have to consider two cases: $|\gamma|=1$ and $|\gamma|=2$. 
Case 1: First assume that $|\gamma|=1$. Then, $\gamma=\left(\gamma_{0}\right)$ and

$$
w=\operatorname{Sw}\left(\gamma_{0}\right)=a^{\gamma_{0}} b \quad \text { and } \quad|w|=\gamma_{0}+1 .
$$

There is one run for $\gamma_{0}>1$, no run for $\gamma_{0}=1$ and obviously $\rho(w)<\frac{4}{5}|w|$.

Case 2: Assume now that $|\gamma|=2$. We have $\gamma=\left(\gamma_{0}, \gamma_{1}\right)$ and

$$
w=\operatorname{Sw}\left(\gamma_{0}, \gamma_{1}\right)=\left(a^{\gamma_{0}} b\right)^{\gamma_{1}} a \quad \text { and } \quad|w|=\left(\gamma_{0}+1\right) \gamma_{1}+1 .
$$

The number of runs in $w$ depends on the values of $\gamma_{0}$ and $\gamma_{1}$ as follows:

$$
\rho(w)=\left\{\begin{array}{cc}
0 & \text { for } \quad \gamma_{0}=1, \gamma_{1}=1 \\
1 & \text { for } \quad \gamma_{0}>1, \gamma_{1}=1 \\
1 & \text { for } \quad \gamma_{0}=1, \gamma_{1}>1 \\
\gamma_{1}+1 & \text { for } \quad \gamma_{0}>1, \gamma_{1}>1
\end{array} .\right.
$$

In each case we have

$$
\rho(w)<\frac{4}{5}\left(\left(\gamma_{0}+1\right) \gamma_{1}+1\right)=\frac{4}{5}|w|
$$

and the proof is complete.

Now we are ready to estimate the asymptotic bound for the number of runs in all standard Sturmian words.

Theorem 16 (Upper bound).

For each standard word $w$ we have $\rho(w) \leqslant \frac{4}{5}|w|$.

Proof. Let $\gamma=\left(\gamma_{0}, \ldots, \gamma_{n}\right)$ be a directive sequence and $w=\operatorname{Sw}\left(\gamma_{0}, \ldots, \gamma_{n}\right)$ be a standard word. Recall the formula (5) from Theorem 11 and observe that $\Delta(\gamma) \leqslant 0$.

The case of $n \leqslant 2$ follows from Lemma 15. It is sufficient to prove the statement for $n \geqslant 3$. We consider four cases depending on the values of $\gamma_{0}$ and $\gamma_{1}$.

Case 1: $\gamma_{0}=\gamma_{1}=1$.

We have, due to Lemma 10 and equation (5):

$$
|w|=3 A+2 B \quad \text { and } \quad \rho(w) \leqslant 2 A+2 B .
$$

Hence

$$
\frac{\rho(w)}{|w|} \leqslant \frac{2 A+2 B}{3 A+2 B} \leqslant \frac{4}{5},
$$

due to inequalities $A \geqslant B \geqslant 1$. This completes the proof of this case.

Case 2: $\gamma_{0}=1 ; \gamma_{1}>1$.

We have, due to Lemma 10 and equation (5):

$$
|w|=\left(2 \gamma_{1}+1\right) A+2 B \quad \text { and } \quad \rho(w) \leqslant\left(\gamma_{1}+2\right) A+B .
$$


Consequently:

$$
\frac{\rho(w)}{|w|} \leqslant \frac{\left(\gamma_{1}+2\right) A+B}{\left(2 \gamma_{1}+1\right) A+2 B} \leqslant \frac{4}{5},
$$

because $\gamma_{1} \geqslant 2$ and $\frac{\gamma_{1}+2}{2 \gamma_{1}+1} \leqslant \frac{4}{5}$.

Case 3: $\gamma_{0}>1 ; \gamma_{1}=1$.

Due to equation (5) and Lemma 10, we have:

$$
\begin{gathered}
\rho(w) \leqslant 2 A+3 B \\
|w|=\left(\left(\gamma_{0}+2\right) A+\left(\gamma_{0}+1\right) B\right) \geqslant 4 A+3 B,
\end{gathered}
$$

and consequently:

$$
\frac{\rho(w)}{|w|} \leqslant \frac{2 A+3 B}{4 A+3 B} \leqslant \frac{3 A+2 B}{4 A+3 B} \leqslant \frac{3}{4}<\frac{4}{5} .
$$

Case $4: \gamma_{0}>1 ; \gamma_{1}>1$.

In this case, due to equation (5) and Lemma 10, we have:

$$
\begin{gathered}
\rho(w) \leqslant\left(2 \gamma_{1}+1\right) A+2 B \\
|w|=\left(\left(\gamma_{0}+1\right) \gamma_{1}+1\right) A+\left(\gamma_{0}+1\right) B,
\end{gathered}
$$

and consequently

$$
\frac{\rho(w)}{|w|} \leqslant \frac{\left(2 \gamma_{1}+1\right) A+2 B}{\left(\left(\gamma_{0}+1\right) \gamma_{1}+1\right) A+\left(\gamma_{0}+1\right) B} \leqslant \frac{\left(2 \gamma_{1}+1\right) A+2 B}{\left(3 \gamma_{1}+1\right) A+3 B} \leqslant \frac{4}{5},
$$

because

$$
\frac{2 \gamma_{1}+1}{3 \gamma_{1}+1} \leqslant \frac{4}{5}
$$

This completes the proof of the theorem.

The above results give an asymptotic bound for the number of runs in standard words. Below we construct a strictly growing sequence of standard words to show that this estimation is tight.

\section{Theorem 17.}

For the class $\mathcal{S}$ of standard words we have:

$$
\sup \left\{\frac{\rho(w)}{|w|}: w \in \mathcal{S}\right\}=0.8
$$


Proof. Let $\gamma=(1,2, k, k)$ and $w_{k}=\operatorname{Sw}(1,2, k, k)$. By definition we have

$$
w_{k}=\left((a b a b a)^{k} a b\right)^{k} a b a b a \quad \text { and } \quad\left|w_{k}\right|=5 k^{2}+2 k+5,
$$

and due to Equation (5):

$$
\left|\rho\left(w_{k}\right)\right|=4 k^{2}-k+3
$$

see Figure 2 for the case $k=3$. Consequently

$$
\lim _{k \rightarrow \infty} \frac{\rho\left(w_{k}\right)}{\left|w_{k}\right|}=\lim _{k \rightarrow \infty} \frac{4 k^{2}-k+3}{5 k^{2}+2 k+5}=0.8
$$

and this completes the proof.

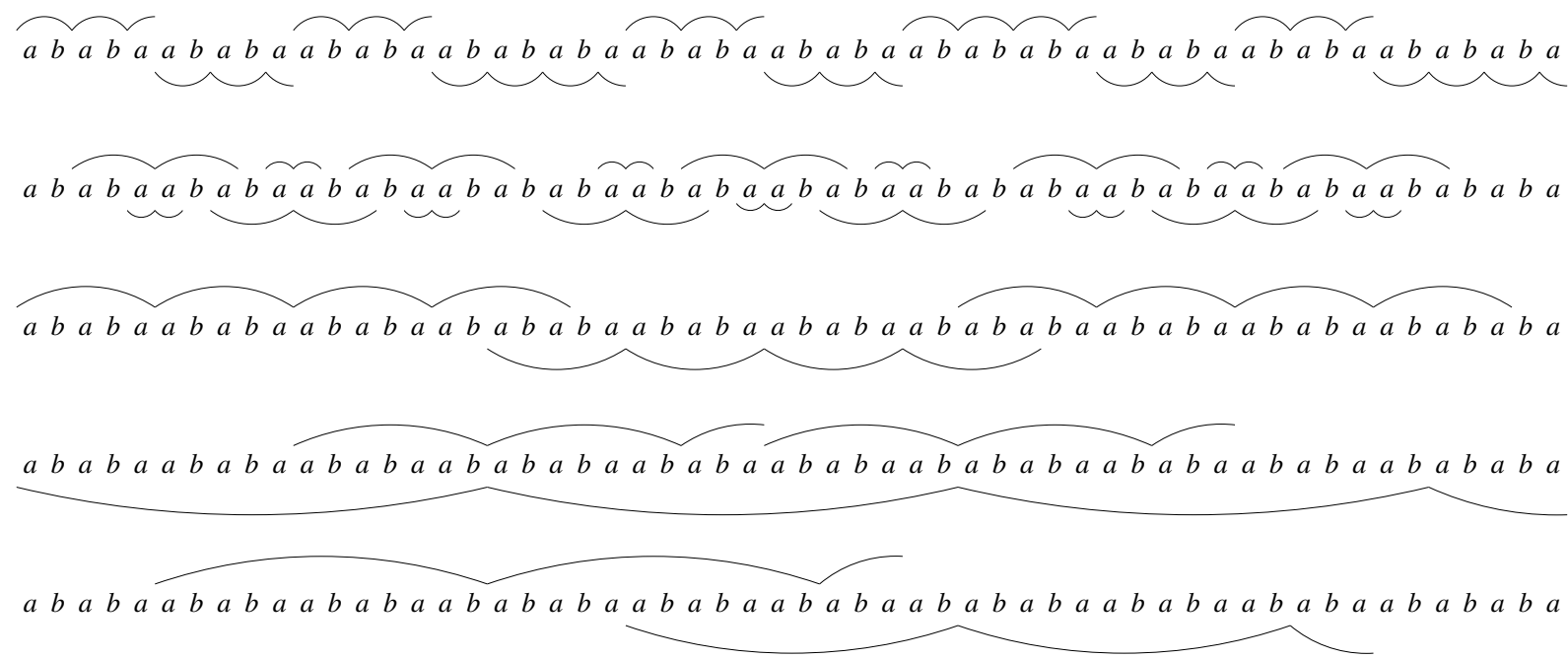

Figure 2: The structure of runs in the standard word $\mathrm{Sw}(1,2, k, k)$ for $k=3$. There are $4 k^{2}-k+3=36$ runs.

\section{The proof of Theorem 11 (the formula)}

This section is devoted to the proof of Theorem 11. We begin with a characterization of the structure of possible periods of maximal repetitions in standard words. Recall the recurrent definition given by equation (1) and the words $x_{i}$ defined there.

The following lemma is a version of Theorem 1 in [5] using slightly different notation.

Lemma 18 (Structural Lemma).

The period of each maximal repetition in the standard word $\operatorname{Sw}\left(\gamma_{0}, \gamma_{1}, \ldots, \gamma_{n}\right)$ is of the form $\left(x_{i}\right)^{j} x_{i-1}$, where $0 \leqslant j<\gamma_{i}$. 
To prove the above lemma it is sufficient to show that no factor of the word $\operatorname{Sw}\left(\gamma_{0}, \ldots, \gamma_{n}\right)$ that does not satisfy the condition given there could be the generator of some repetition, see the proof of Theorem 1 in [5] for more details.

The main idea of the proof of Theorem 11 is a partition of the set of all maximal repetitions in the word $\operatorname{Sw}\left(\gamma_{0}, \ldots, \gamma_{n}\right)$ into three separate categories depending on the length of their periods. We say that a run is:

short - if the length of its period does not exceed $\left|x_{1}\right|$,

large - if the length of its period exceeds $\left|x_{2}\right|$,

medium - otherwise.

Denote by $\rho_{S}(w), \rho_{M}(w)$ and $\rho_{L}(w)$ the number of short, medium and large runs in the word $w$. respectively.

Example 19. Recall the word $w=\operatorname{Sw}(1,2,1,3,1)$ from Example 1 and the set of its maximal repetitions. In this case we have:

- 10 short runs (the periods are $a$ and $a b$ ),

- 8 medium runs (the periods are $a b a$ and $a b a b a$ ),

- 1 large run (the period is ababaab),

see Figure 1 for comparison.

\section{Counting short runs}

We start with the computation of the number of short runs. These are runs with the periods of the form $a$ or $a^{+} b$. Their number depends on the value of $\gamma_{0}$ and $\gamma_{1}$.

Lemma 20 (Short Runs).

Let $w=\operatorname{Sw}\left(\gamma_{0}, \ldots, \gamma_{n}\right)$ be a standard word. The number of short runs in $w$ is given by the formula:

$$
\rho_{S}(w)=\left\{\begin{array}{ll}
N_{\gamma}(2)+N_{\gamma}(3)-1 & \text { for } \gamma_{0}=1, \gamma_{1}=1 \\
2 N_{\gamma}(2)-\operatorname{odd}(n) & \text { for } \quad \gamma_{0}=1, \gamma_{1}>1 \\
N_{\gamma}(1)+N_{\gamma}(3)-\operatorname{even}(n) & \text { for } \quad \gamma_{0}>1, \gamma_{1}=1 \\
N_{\gamma}(1)+N_{\gamma}(2) & \text { for } \quad \gamma_{0}>1, \gamma_{1}>1
\end{array} .\right.
$$

Proof. Short runs are maximal repetitions with periods of the form $a$ or $a^{k} b$. We estimate the number of runs with periods of each type separately. 
Case 1: runs with periods of the form $a$.

First assume that $\gamma_{0}>0$. Every run with the period $a$ in $\operatorname{Sw}\left(\gamma_{0}, \ldots, \gamma_{n}\right)$ equals $a^{\gamma_{0}}$ or $a^{\gamma_{0}+1}$ and is followed by the single letter $b$. Due to Lemma 4 , every such run in $\operatorname{Sw}\left(\gamma_{0}, \ldots, \gamma_{n}\right)$ corresponds to the letter $a$ in $\operatorname{Sw}\left(\gamma_{1}, \ldots, \gamma_{n}\right)$. Hence in this case we have $N_{\gamma}(1)$ runs with the period $a$.

Assume now that $\gamma_{0}=1$. In this case the word $\operatorname{Sw}\left(\gamma_{0}, \ldots, \gamma_{n}\right)$ consists of the blocks of two types: $a b$ or $a a b$ and only the blocks of the second type include the runs with the period $a$. Due to Lemma 4 every such run in $\operatorname{Sw}\left(\gamma_{0}, \ldots, \gamma_{n}\right)$ corresponds to the letter $b$ followed by the letter $a$ in $\operatorname{Sw}\left(\gamma_{1}, \ldots, \gamma_{n}\right)$, hence the number of such runs equals the number of blocks ba in $\operatorname{Sw}\left(\gamma_{1}, \ldots, \gamma_{n}\right)$.

Recall that for an even length of the directive sequence $\left|\left(\gamma_{1}, \ldots, \gamma_{n}\right)\right|(n$ is even) the word $\operatorname{Sw}\left(\gamma_{1}, \ldots, \gamma_{n}\right)$ ends with $b a$ and in this case the number of runs with the period $a$ in in $\operatorname{Sw}\left(\gamma_{1}, \ldots, \gamma_{n}\right)$ equals the number of the letters $b$ in $\operatorname{Sw}\left(\gamma_{1}, \ldots, \gamma_{n}\right)$, which is $N_{\gamma}(2)$. For an odd length of the directive sequence $\left|\left(\gamma_{1}, \ldots, \gamma_{n}\right)\right|(n$ is odd $)$ the word $\operatorname{Sw}\left(\gamma_{1}, \ldots, \gamma_{n}\right)$ ends with $a b$ and the last letter $b$ does not correspond to a run in $\operatorname{Sw}\left(\gamma_{0}, \ldots, \gamma_{n}\right)$. In this case, the number of runs with the period $a$ in $\operatorname{Sw}\left(\gamma_{0}, \ldots, \gamma_{n}\right)$ is one less than the number of the letters $b$ in $\operatorname{Sw}\left(\gamma_{1}, \ldots, \gamma_{n}\right)$, which is $N_{\gamma}(2)-1$. Finally, the above reasoning can be summarized as:

$$
\left\{\begin{array}{ll}
N_{\gamma}(2)-\operatorname{odd}(n) & \text { for } \gamma_{0}=1 \\
N_{\gamma}(1) & \text { for } \gamma_{0}>1
\end{array} .\right.
$$

Case 2: runs with periods of the form $a^{k} b$.

Notice that, due to Equation (2) and Lemma 4, the runs with the periods $a^{\gamma_{0}} b$ and $a^{\gamma_{0}+1} b$ in the word $\operatorname{Sw}\left(\gamma_{0}, \ldots, \gamma_{n}\right)$ correspond to the runs with the periods $a$ in the word $\operatorname{Sw}\left(\gamma_{1}, \ldots, \gamma_{n}\right)$. Similar argumentation as above shows that the number of such runs in the word $\operatorname{Sw}\left(\gamma_{0}, \ldots, \gamma_{n}\right)$ equals:

$$
\left\{\begin{array}{ll}
N_{\gamma}(3)-\operatorname{even}(n) & \text { for } \gamma_{1}=1 \\
N_{\gamma}(2) & \text { for } \gamma_{1}>1
\end{array} .\right.
$$

Combining the results from the two above cases we conclude the proof of the lemma.

\section{Counting medium runs}

Recall that medium runs are maximal repetitions with periods $\left(x_{1}\right)^{k} x_{0}$ for $0<k<\gamma_{1}$ and $x_{2}$, where $x_{i}$ are as in Equation (1). Observe that medium runs appear in standard words generated by directive sequences of the length at least 3 . We have to consider two cases: the directive sequences of the length 3 and the longer ones. The value of $\gamma_{0}$ does not affect the number of medium runs, hence to simplify calculations we assume in further proofs that $\gamma_{0}=1$. We start with counting medium runs in standard words generated by directive sequences of the length greater than 3 . 
Lemma 21 (Medium runs, $n \geqslant 3$ ).

Let $w=\operatorname{Sw}\left(\gamma_{0}, \ldots, \gamma_{n}\right)$ be a standard word and $n \geqslant 3$. The number of medium runs in $w$ is given by the formula:

$$
\rho_{M}(w)=N_{\gamma}(1)-N_{\gamma}(2)-\gamma_{1}+1 .
$$

The statement of Lemma 21 is corollary to the following stronger claim:

Claim 22. Let $w=\operatorname{Sw}\left(\gamma_{0}, \ldots, \gamma_{n}\right)$ be a standard word. There are:

(1) $N_{\gamma}(2)-1$ runs with the period $\left(x_{1}\right)^{i} x_{0}$ for each $0<i<\gamma_{1}$.

(2) $N_{\gamma}(3)$ runs with the period $x_{2}$.

Proof. Point (1)

The word $\operatorname{Sw}\left(\gamma_{0}, \ldots, \gamma_{n}\right)$ has the form:

$$
(a b)^{\alpha_{1}} a(a b)^{\alpha_{2}} a \ldots(a b)^{\alpha_{s}} a a b \quad \text { or } \quad(a b)^{\alpha_{1}} a(a b)^{\alpha_{2}} a \ldots(a b)^{\alpha_{s}} a,
$$

where $0<\alpha_{i} \leqslant\left(\gamma_{1}+1\right)$ and $s=N_{\gamma}(2)$, because (due to Lemma 4) every factor $(a b)^{\alpha_{i}} a$ in $\operatorname{Sw}\left(\gamma_{0}, \ldots, \gamma_{n}\right)$ corresponds to the letter $a$ in $\operatorname{Sw}\left(\gamma_{2}, \ldots, \gamma_{n}\right)$. For example, see Figure 3, the word $\operatorname{Sw}(1,4,2,2)$ has the form

$$
\operatorname{Sw}(1,4,2,2)=(a b)^{4} a(a b)^{4} a(a b)^{5} a(a b)^{4} a(a b)^{5} a .
$$

Each pair of neighboring factors: $(a b)^{\alpha_{i}} a \cdot(a b)^{\alpha_{i+1}} a$ produces $\gamma_{1}-1$ runs with the period $(a b)^{k} a$ for each $0<k<\gamma_{1}$. In the word $\operatorname{Sw}\left(\gamma_{0}, \ldots, \gamma_{n}\right)$ we have $N_{\gamma}(2)-1$ such pairs and therefore $\left(N_{\gamma}(2)-1\right)\left(\gamma_{1}-1\right)$ medium runs with the periods $\left(x_{1}\right)^{k} x_{0}$ for $0<k<\gamma_{1}$.

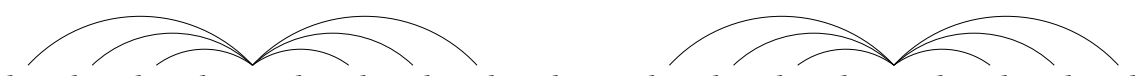

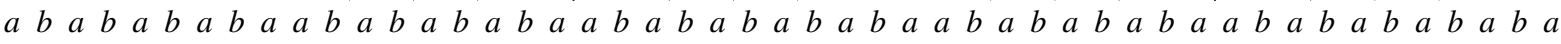

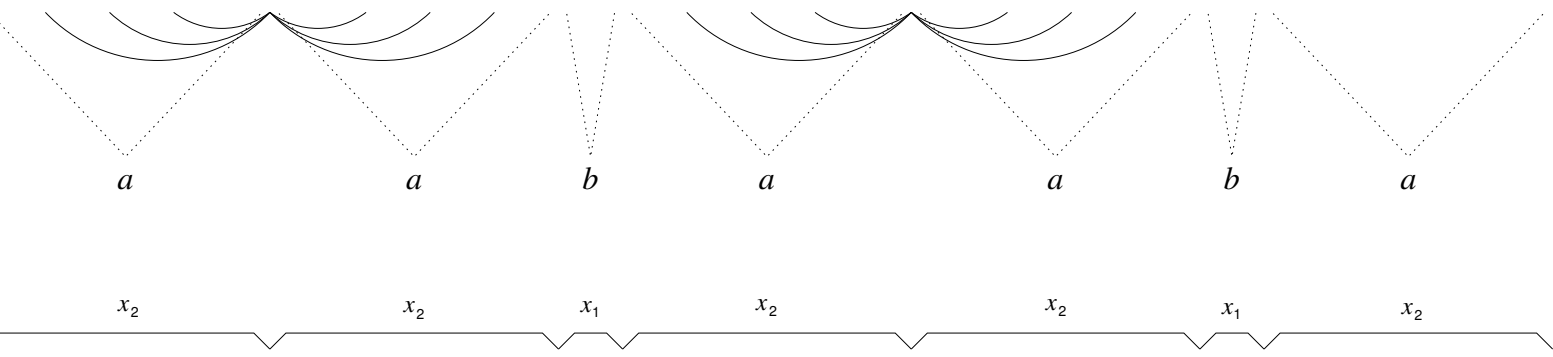

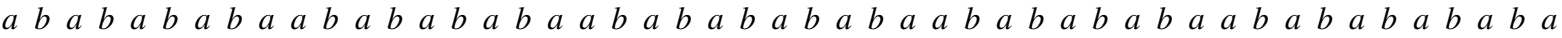

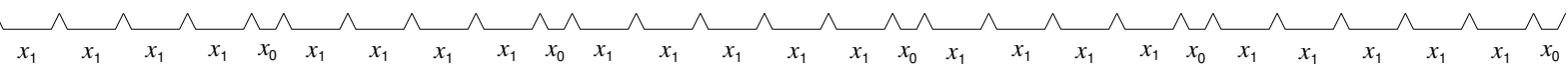

Figure 3: The structure of runs with the periods $\left|x_{1}\right|<p<\left|x_{2}\right|$ for the word $\operatorname{Sw}(1,4,2,2)$ and its decomposition into words $x_{1}, x_{2}$ and $x_{0}, x_{1}$. 


\section{Point (2)}

The word $\operatorname{Sw}\left(\gamma_{0}, \ldots, \gamma_{n}\right)$ can be represented as a sequence of concatenated words $x_{1}$ and $x_{2}$ and has the form:

$$
(a): x_{2}^{\alpha_{1}} x_{1} x_{2}^{\alpha_{2}} x_{1} \ldots x_{2}^{\alpha_{s}} x_{1} x_{2} \quad \text { or } \quad(b): x_{2}^{\beta_{1}} x_{1} x_{2}^{\beta_{2}} x_{1} \ldots x_{2}^{\beta_{s}} x_{1} \text {. }
$$

For example the word $\operatorname{Sw}(1,4,2,2)$ has the decomposition $x_{2}^{2} x_{1} x_{2}^{2} x_{1} x_{2}$, see Figure 3 .

First assume the case $(a)$. Each run with the period $x_{2}$ has the form $\left(x_{2}\right)^{k} x_{1}$. By the definition of standard words, the factor $x_{1} x_{2}$ has $x_{2}$ as a prefix. Therefore, the number of such runs in $\operatorname{Sw}\left(\gamma_{0}, \ldots, \gamma_{n}\right)$ equals the number of factors $x_{1}$ in the decomposition mentioned above, which (due to Lemma 4) corresponds to the number of the letters $b$ in $\operatorname{Sw}\left(\gamma_{2}, \ldots, \gamma_{n}\right)$, namely $N_{\gamma}(3)$.

Assume now the case $(b)$. The word $\operatorname{Sw}\left(\gamma_{0}, \ldots, \gamma_{n}\right)$ has the suffix $x_{1}$ but in this case we have $\beta_{s} \geqslant 2$. Hence the number of runs with the period $x_{2}$ is the same as in the previous case.

Proof of Lemma 21. Summing up the formulas from the points (1) and (2) of Claim 22 we obtain:

$$
\begin{aligned}
\rho_{M} & =\left(N_{\gamma}(2)-1\right)\left(\gamma_{1}-1\right)+N_{\gamma}(3) \\
& =\left(\gamma_{1} N_{\gamma}(2)+N_{\gamma}(3)\right)-N_{\gamma}(2)-\gamma_{1}+1 \\
& =N_{\gamma}(1)-N_{\gamma}(2)-\gamma_{1}+1
\end{aligned}
$$

and this completes the proof of the lemma.

The structure of the medium runs in standard words defined by directive sequences of the length 3 is slightly different.

Lemma 23 (Medium runs, $\mathrm{n}=2$ ).

Let $w=\operatorname{Sw}\left(\gamma_{0}, \gamma_{1}, \gamma_{2}\right)$ be a standard word. The number of medium runs in $w$ is given by the formula:

$$
\rho_{M}(w)=N_{\gamma}(1)-N_{\gamma}(2)-\gamma_{1}+1-\operatorname{unary}\left(\gamma_{2}\right)
$$

Proof. The proof of the case $\gamma_{2}>1$ uses the same argumentation as the proof of Lemma 21.

In the case $\gamma_{2}=1$, the word $\operatorname{Sw}\left(\gamma_{0}, \gamma_{1}, \gamma_{2}\right)$ has the decomposition

$$
\operatorname{Sw}\left(\gamma_{0}, \gamma_{1}, \gamma_{2}\right)=\left(a^{\gamma_{0}} b\right) a \cdot a^{\gamma_{0}} b=x_{2} \cdot x_{1}
$$

There is no run with the period $x_{2}$, and we have to subtract 1 from the number of the factors $x_{1}$ in this case. 


\section{The recurrence for large runs}

Recall that the run is called large if it has the period of the length greater than $\left|x_{2}\right|$, where $x_{2}$ is as in Equation (1). We reduce the problem of counting large runs to counting medium runs, using the morphic representation of standard words.

Let $h$ be a morphism and let $v=a_{1} a_{2} \ldots a_{k}$ be the word of the length $k$. The morphism $h$ defines the partition of the word $w=h(v)$ into segments $h\left(a_{1}\right), h\left(a_{2}\right), \ldots, h\left(a_{t}\right)$. These segments are called the $h$-blocks. We say that a factor $x$ of the word $w$ is synchronized with the morphism $h$ in $w$ if and only if each occurrence of $x$ in $w$ starts at the beginning of some $h$-block and ends at the end of some $h$-block. Observe that every factor in $w$ that is synchronized with $h$ corresponds to some factor in $v$, hence the morphism $h$ preserves the structure of the factors that are synchronized with it.

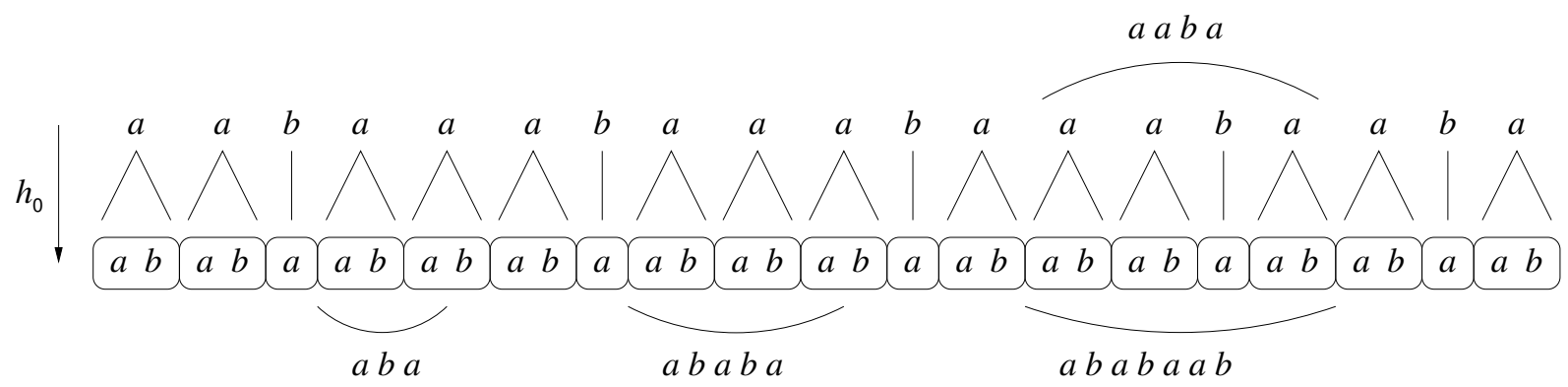

Figure 4: The periods of the medium runs $x_{1} x_{0}=a b a$ and $x_{2}=$ ababa do not synchronize with the morphism $h_{0}$ in the word $\mathrm{Sw}(1,2,1,3,1)$, while the period of the large run $x_{3}=a b a b a a b$ is synchronized with $h_{0}$ and corresponds to the medium run with the period $x_{1} x_{0}=$ aaba in the word $\operatorname{Sw}(2,1,3,1)$.

Example 24. Let $w=\operatorname{Sw}(1,2,1,3,1)$ and $v=\operatorname{Sw}(2,1,3,1)$ be standard words and $h_{0}$ be the morphism defined as:

$$
h_{0}:\left\{\begin{array}{l}
a \longrightarrow a b \\
b \longrightarrow a
\end{array} .\right.
$$

Recall that

$$
\begin{aligned}
\operatorname{Sw}(1,2,1,3,1) & =h_{0}(\operatorname{Sw}(2,1,3,1)), \\
\operatorname{Sw}(1,2,1,3,1) & =\text { ababaabababaabababaabababaababaab, } \\
\operatorname{Sw}(2,1,3,1) & =\text { aabaaabaaabaaabaaba. }
\end{aligned}
$$

The factors $w[6 . .8]=a b a$ and $w[13 . .17]=a b a a b$ are not synchronized with the morphism $h_{0}$, because both of them ends in the middle some $h_{0}$-block. The factor $w[22 . .28]$ starts at the beginning of some $h_{0}$-block and ends at the end of some $h_{0}$-block, hence is synchronized with the morphism $h_{0}$. Moreover it corresponds to the factor $v[13 . .16]=a a b a$, see Figure 4 for comparison. 
Lemma 25 (Synchronization Lemma).

The periods of large runs in the word $\operatorname{Sw}\left(\gamma_{0}, \ldots, \gamma_{n}\right)$ are synchronized with the morphism $h_{0}$.

Proof. Let $h_{0}$ be the morphism defined as

$$
h_{0}:\left\{\begin{array}{ll}
a & \longrightarrow a^{\gamma_{0}} b \\
b & \longrightarrow a
\end{array} .\right.
$$

Due to Lemma 4 we have

$$
\operatorname{Sw}\left(\gamma_{0}, \ldots, \gamma_{n}\right)=h_{0}\left(\operatorname{Sw}\left(\gamma_{1}, \ldots, \gamma_{n}\right)\right) .
$$

Moreover, $h_{0}$ determines the partition of $\operatorname{Sw}\left(\gamma_{0}, \ldots, \gamma_{n}\right)$ into $h_{0}$-blocks of the form $a^{\gamma_{0}} b$ and $a$, see Figure 4 for the partition of $\operatorname{Sw}(1,2,1,3,1)$.

Recall that the period of each large run in the word $\operatorname{Sw}\left(\gamma_{0}, \ldots, \gamma_{n}\right)$ is of the form $\left(x_{i}\right)^{k} x_{i-1}$, where $0 \leqslant k<\gamma_{i}$ and $i \geqslant 2$. By the definition of standard words the factor $\left(x_{i}\right)^{k} x_{i-1}$ starts with $a^{\gamma_{0}} b$, hence at the beginning of some $h_{0}$-block.

For even $i \geqslant 2$, the subword $\left(x_{i}\right)^{k} x_{i-1}$ ends with $x_{1}=a^{\gamma_{0}} b$, hence at the end of some $h_{0}$-block, and is obviously synchronized with $h_{0}$.

For odd $i \geqslant 2$ the factor $\left(x_{i}\right)^{k} x_{i-1}$ ends with

$$
x_{3} \cdot x_{2}=x_{2}^{\gamma_{2}} x_{1} \cdot x_{1}^{\gamma_{1}} x_{0}=x_{2}^{\gamma_{2}} \cdot\left(a^{\gamma_{0}} b\right)^{\gamma_{1}+1} a .
$$

First assume that $\left(x_{i}\right)^{k} x_{i-1}$ is followed by the block $a^{\gamma_{0}} b$. The single letter $a$ at the end of $\left(x_{i}\right)^{k} x_{i-1}$ is then the whole $h_{0}$-block and $\left(x_{i}\right)^{k} x_{i-1}$ is synchronized with the morphism $h_{0}$.

Assume now that $\left(x_{i}\right)^{k} x_{i-1}$ ends with $\left(a^{\gamma_{0}} b\right)^{\gamma_{1}+1} a$ and is followed by $\left(a^{\gamma_{0}-1} b\right)$, namely it ends in the middle of some $h_{0}$-block. In this case we have the occurrence of the factor $\left(a^{\gamma_{0}} b\right)^{\gamma_{1}+2}$ in $\operatorname{Sw}\left(\gamma_{0}, \ldots, \gamma_{n}\right)$, which is reduced by the morphism $h_{0}^{-1}$ to the factor $a^{\gamma_{1}+2} b$ in $\operatorname{Sw}\left(\gamma_{1}, \ldots, \gamma_{n}\right)$. By the definition standard words, $\operatorname{Sw}\left(\gamma_{1}, \ldots, \gamma_{n}\right)$ can include only blocks of two types: the short block $-a^{\gamma_{1}} b$ and the long block $-a^{\gamma_{1}+1} b$, hence we have the contradiction and the proof is complete.

The following lemma, which is a direct corollary to Synchronization lemma, allows to reduce the problem of counting the large runs in the word $\operatorname{Sw}\left(\gamma_{0}, \ldots, \gamma_{n}\right)$ to those in $\operatorname{Sw}\left(\gamma_{1}, \ldots, \gamma_{n}\right)$.

Lemma 26 (Recurrence Lemma). Let $w=\operatorname{Sw}\left(\gamma_{0}, \ldots, \gamma_{n}\right)$ and $v=\operatorname{Sw}\left(\gamma_{1}, \ldots, \gamma_{n}\right)$ be standard words. The number of large runs in $w$ is given by the recurrence

$$
\rho_{L}(w)=\rho_{L}(v)+\rho_{M}(v) .
$$


Proof. The synchronization lemma implies that the morphism defined as in Equation (2) preserve the structure of long runs in standard words. Recall that $\operatorname{Sw}\left(\gamma_{0}, \ldots, \gamma_{n}\right)$ is reduced by $h_{0}^{-1}$ to $\operatorname{Sw}\left(\gamma_{1}, \ldots, \gamma_{n}\right)$. Therefore, every large run $\alpha$ in $\operatorname{Sw}\left(\gamma_{0}, \ldots, \gamma_{n}\right)$ corresponds to some run $\beta$ in $\operatorname{Sw}\left(\gamma_{1}, \ldots, \gamma_{n}\right)$.

Due to Lemma 18, the period of the run $\alpha$ is of the form $\left(x_{i}\right)^{k} x_{-1}$, where $0<k \leqslant \gamma_{i}$ and $i \geqslant 2$. The corresponding run $\beta$ is either large (for $i=2$ ) or medium (for $i=2$ ). Hence, to compute all large runs in $\operatorname{Sw}\left(\gamma_{0}, \ldots, \gamma_{n}\right)$ it is sufficient to compute all large and medium runs in $\operatorname{Sw}\left(\gamma_{1}, \ldots, \gamma_{n}\right)$.

The statement of the next lemma gives the compact formula for the number of the medium and the large runs in standard words.

Lemma 27 (Large Runs).

Let $w=\operatorname{Sw}\left(\gamma_{0}, \ldots, \gamma_{n}\right)$ be a standard word. We have

$$
\rho_{L}(w)+\rho_{M}(w)=N_{\gamma}(1)+n-1-\left(\gamma_{1}+\ldots+\gamma_{n}\right)-\operatorname{unary}\left(\gamma_{n}\right) .
$$

Proof. Due to the formulas from Lemma 21 and Lemma 23 and the recurrence from Lemma 26 we have

$$
\begin{aligned}
\rho_{L}(w)+\rho_{M}(w)= & \sum_{i=0}^{n-2} \rho_{M}\left(\operatorname{Sw}\left(\gamma_{i}, \ldots, \gamma_{n}\right)\right) \\
= & \left(N_{\gamma}(1)-N_{\gamma}(2)-\gamma_{1}+1\right)+ \\
\vdots & \left(N_{\gamma}(n-2)-N_{\gamma}(n-1)-\gamma_{n-2}+1\right)+ \\
& \left(N_{\gamma}(n-1)-N_{\gamma}(n)-\gamma_{n-1}+1-\operatorname{unary}\left(\gamma_{n}\right)\right) .
\end{aligned}
$$

Taking into account that $N_{\gamma}(n)=\gamma_{n}$ the above formula can be written as

$$
\rho_{L}(w)+\rho_{M}(w)=N_{\gamma}(1)+(n-1)-\left(\gamma_{1}+\ldots+\gamma_{n}\right)-\operatorname{unary}\left(\gamma_{n}\right),
$$

and this concludes the proof.

Now we are ready to prove the formula for the number of runs in standard words given by Equation (5).

Proof of Theorem 11. Let $w=\operatorname{Sw}\left(\gamma_{0}, \ldots, \gamma_{n}\right)$ be a standard word. Recall that we divide the set of all runs in $w$ into three disjoint subsets of short, medium and large runs, depending on the length of their periods. The number $\rho(w)$ of runs in $w$ equals:

$$
\rho(w)=\rho_{S}(w)+\rho_{M}(w)+\rho_{L}(w)
$$

where $\rho_{S}(w), \rho_{M}(w)$ and $\rho_{L}(w)$ denote the number of short, medium and large runs in $w$. 
Due to Lemma 20, the number of short runs in $w$ is given by the formula:

$$
\rho_{S}(w)=\left\{\begin{array}{ll}
N_{\gamma}(2)+N_{\gamma}(3)-1 & \text { for } \gamma_{0}=1, \gamma_{1}=1 \\
2 N_{\gamma}(2)-\operatorname{odd}(n) & \text { for } \gamma_{0}=1, \gamma_{1}>1 \\
N_{\gamma}(1)+N_{\gamma}(3)-\operatorname{even}(n) & \text { for } \gamma_{0}>1, \gamma_{1}=1 \\
N_{\gamma}(1)+N_{\gamma}(2) & \text { for } \gamma_{0}>1, \gamma_{1}>1
\end{array} .\right.
$$

Moreover, due to Lemma 27, the number of large and medium runs in $w$ is given as:

$$
\rho_{L}(w)+\rho_{M}(w)=N_{\gamma}(1)+n-1-\left(\gamma_{1}+\ldots+\gamma_{n}\right)-\operatorname{unary}\left(\gamma_{n}\right) .
$$

Finally, combining the above formulas, we have:

$$
\rho(w)= \begin{cases}2 A+2 B+\Delta(\gamma)-1 & \text { for } \gamma_{0}=\gamma_{1}=1 \\ \left(\gamma_{1}+2\right) A+B+\Delta(\gamma)-\operatorname{odd}(n) & \text { for } \gamma_{0}=1 ; \gamma_{1}>1 \\ 2 A+3 B+\Delta(\gamma)-\operatorname{even}(n) & \text { for } \gamma_{0}>1 ; \gamma_{1}=1 \\ \left(2 \gamma_{1}+1\right) A+2 B+\Delta(\gamma) & \text { for } \gamma_{0}>1 ; \gamma_{1}>1\end{cases}
$$

where:

$$
\begin{aligned}
A & =N_{\gamma}(2)=\left|\operatorname{Sw}\left(\gamma_{2}, \gamma_{3}, \ldots, \gamma_{n}\right)\right|_{a}, \\
B & =N_{\gamma}(3)=\left|\operatorname{Sw}\left(\gamma_{3}, \gamma_{4}, \ldots, \gamma_{n}\right)\right|_{a}, \\
\Delta(\gamma) & =n-1-\left(\gamma_{1}+\ldots+\gamma_{n}\right)-\operatorname{unary}\left(\gamma_{n}\right) .
\end{aligned}
$$

This completes the proof of the theorem.

\section{$7 \quad$ Final remarks}

The aim of this paper was to study problems related to maximal repetitions for one of the most thoroughly investigated class of strings in combinatorics on words - the standard Sturmian words. We have presented formulas for the numbers of runs along with the detailed analysis of their asymptotic behaviour. The complete understanding of their combinatorial structure for a large class of complicated words is a step towards a better understanding of this problem in general.

The maximal repetition ratio 0.8 for standard words has been first discovered by us during computer experiments with very long strings. Similarly, we were tuning many intermediate formulas with the assistance of the computer. Our algorithm for computing the number of runs in standard words is an example of a very fast computation on highly compressed texts in linear time with respect to the size of their compressed representation. 


\begin{tabular}{|r||r|l|l||r|l|l|}
\hline \multicolumn{1}{|c||}{$\mathrm{n}$} & \multicolumn{3}{|c||}{ All binary words } & \multicolumn{3}{|c|}{ Standard words } \\
\cline { 2 - 6 } & $\rho(n)$ & $\rho(n) / n$ & Example word & 1 & 0.2 & ababa \\
\hline 5 & 2 & 0.4 & aaabb & 1 & 0.1667 & aaaaba \\
7 & 3 & 0.5 & aabaab & 3 & 0.4286 & aabaaba \\
8 & 4 & 0.5714 & aabaabb & 3 & 0.375 & abaababa \\
9 & 5 & 0.625 & aabbaabb & 3 & 0.3333 & aaabaaaba \\
10 & 6 & 0.5555 & aaabbaabb & 4 & 0.4 & aabaabaaba \\
11 & 7 & 0.6 & aabaabbaab & 5 & 0.4545 & aabaaabaaba \\
12 & 8 & 0.6667 & aabaabbaabb & 6 & 0.5 & ababaababaab \\
13 & 8 & 0.6154 & aaabaabbaabaaa & 7 & 0.5385 & abaababaababa \\
14 & 10 & 0,7143 & aabaabbaabaabb & 5 & 0.3571 & abaabaabaababa \\
15 & 10 & 0.6667 & aaabaabbaabaabb & 6 & 0.4 & aabaaabaaabaaba \\
16 & 11 & 0.6875 & aabaabbaabaabbaa & 7 & 0.4375 & abababaabababaab \\
17 & 12 & 0.7059 & aabaababbabaababb & 9 & 0.5294 & ababaababaababaab \\
18 & 13 & 0.7222 & aabaabbaabaabbaabb & 10 & 0.5556 & aabaaabaabaaabaaba \\
19 & 14 & 0.7368 & aabaabbaabaabbaabaa & 1 & 0.5789 & ababaabababaababaab \\
20 & 15 & 0.75 & aababaababbabaababaa & 13 & 0.4 & ababababaababababaab \\
21 & 15 & 0.7143 & aaababaababbabaababaa & 12 & 0.545 & abaababaabaababaababa \\
22 & 16 & 0.7273 & aabaababaababbabaababb & 13 & 0.5652 & ababaababaababaababaab \\
23 & 17 & 0.7391 & aabaababaababbabaababaa & 13 & 0.5417 & aabaabaaabaabaaabaabaaba \\
24 & 18 & 0.75 & aabaabbaabaabbabbaabbabb & 14 & 0.56 & aabaaabaabaaabaabaaabaaba \\
25 & 19 & 0.76 & aabaabbaabaaabaabbaabaabb & 15 & 0.5769 & ababaabababaabababaababaab \\
26 & 20 & 0.7692 & aababaababbabaababaababbab & 16 & 0.5926 & aabaabaaabaabaabaaabaabaaba \\
27 & 21 & 0.7778 & aabaababaababbabaababaababb & 16 & 0.5714 & abaababaababaababaababaabaab \\
28 & 22 & 0.7857 & aababaababbabaababaababbabaa & 18 & 0.6207 & ababaababaabababaababaababaab \\
29 & 23 & 0.7931 & aababaababbabaababaababbababb & 18 & 0.6 & abaabaababaabaabaababaabaabaab \\
30 & 24 & 0.8 & aababbabaababbababbabaababbaba & 20 & 0.6452 & ababaabababaababaabababaababaab \\
31 & 25 & 0.8065 & aababaababbabaababaababbabaabab \\
\hline
\end{tabular}

Table 1: The comparison of the maximal number of runs and the repetition ratio for the class of all binary words and the class of standard words of a given length.

\section{References}

[1] P. Baturo, M. Piątkowski, and W. Rytter. Usefulness of directed acyclic subword graphs in problems related to standard Sturmian words. International Journal of Foundations of Computer Science, 20(6):1005-1023, 2009.

[2] P. Baturo and W. Rytter. Compressed string-matching in standard Sturmian words. Theoretical Computer Science, 410(30-32):2804-2810, 2009.

[3] M. Crochemore and L. Ilie. Analysis of maximal repetitions in strings. In Proceedings of the 32nd International Conference on Mathematical Foundations of Computer Science, volume 4708 of Lecture Notes in Computer Science, pages 465-476. Springer, 2007.

[4] M. Crochemore, L. Ilie, and L. Tinta. Towards a solution of the "runs" conjecture. In Proceedings of the 19th annual symposium on Combinatorial Pattern Matching, volume 5029 of Lecture Notes in Computer Science, pages 290-302. Springer, 2008.

[5] D. Damanik and D. Lenz. Powers in Sturmian sequences. European Journal of Combinatorics, 24(4):377-390, 2003.

[6] F. Franek, A. Karaman, and W. F. Smyth. Repetitions in Sturmian strings. Theoretical Computer Science, 249(2):289-303, 2000. 
[7] F. Franek, R. J. Simpson, and W. F. Smyth. The maximum number of runs in a string. In Proceedings of 14 th Australian Workshop on Combinatiorial Algorithms, pages 26-35, 2003.

[8] F. Franek and Q. Yang. An asymptotic lower bound for the maximal number of runs in a string. Jnternational Journal of Foundations of Computer Science, 19(1):195203, 2008.

[9] C. S. Iliopoulos, D. Moore, and W. F. Smyth. A characterization of the squares in a Fibonacci string. Theoretical Computer Science, 172(1-2):281-291, 1997.

[10] R. Kolpakov and G. Kucherov. Finding maximal repetitions in a word in linear time. In Proceedings of the 40th Annual Symposium on Foundations of Computer Science, pages 596-604. IEEE Computer Society, 1999.

[11] R. M. Kolpakov and G. Kucherov. On maximal repetitions in words. In Proceedings of 12th International Symposium on Fundamentals of Computation Theory, volume 1684 of Lecture Notes in Computer Science, pages 374-385. Springer, 1999.

[12] K. Kusano, W. Matsubara, A. Ishino, H. Bannai, and A. Shinobara. New lower bound for the maximum number of runs in a string. Computing Research Repository, abs/0804.1214, 2008.

[13] M. Piątkowski and W. Rytter. Computing the number of cubic runs in standard Sturmian words. In Proceedings of the 16-th Prague Stringology Conference, pages 106-120. Czech Technical University, 2011.

[14] M. Piątkowski and W. Rytter. Asymptotic behaviour of the maximal number of squares in standard Sturmian words. International Journal of Foundations of Computer Science, 23(2):303-321, 2012.

[15] M. Piątkowski and W. Rytter. The number of cubes in Sturmian words. In Proceedings of the 17-th Prague Stringology Conference, pages 89-102. Czech Technical University, 2012.

[16] N. Pytheas Fogg. Substitutions in Dynamics, Arithmetics and Combinatorics, volume 1794 of Lecture Notes in Mathematics. Springer, 2002.

[17] W. Rytter. The structure of subword graphs and suffix trees of Fibonacci words. Theoretical Computer Science, 363(2):211-223, 2006. 